Canadian

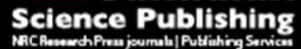

Canadian Journal of Physiology and Pharmacology Revue canadienne de physiologie et pharmacologie

\title{
Esculetin induces apoptosis of SMMC-7721 cells through IGF-1/PI3K/Akt-mediated mitochondrial pathways
}

\begin{tabular}{|r|l|}
\hline Journal: & Canadian Journal of Physiology and Pharmacology \\
\hline Manuscript ID & cjpp-2016-0548.R1 \\
\hline Manuscript Type: & Article \\
\hline Date Submitted by the Author: & $10-$ Nov-2016 \\
\hline Complete List of Authors: & $\begin{array}{l}\text { Li, Juan; The Third Affiliated Hospital of Jinzhou Medical University } \\
\text { Li, Shuang; Taihe District Hospital of Jinzhou City } \\
\text { Wang, Xiuli; The Third Affiliated Hospital of Jinzhou Medical University } \\
\text { Wang, Hongxin; Jinzhou Medical University }\end{array}$ \\
\hline Keyword: & $\begin{array}{l}\text { Esculetin, Hepatocellular carcinoma SMMC-7721 cells, Apoptosis, IGF-1, } \\
\text { Mitochondria }\end{array}$ \\
\hline &
\end{tabular}


Esculetin induces apoptosis of SMMC-7721 cells through IGF-1/PI3K/Akt-mediated mitochondrial pathways Juan $\mathrm{Li}^{\mathrm{a}}$, Shuang $\mathrm{Li}^{\mathrm{b}}$, Xiuli Wang ${ }^{\mathrm{a}}$, Hongxin Wang ${ }^{\mathrm{c} *}$

${ }^{\mathrm{a}}$ Department of Infectious Disease, The Third Affiliated Hospital of Jinzhou Medical University, Jinzhou 121001, China

${ }^{b}$ Department of Anesthesiology, Taihe District Hospital of Jinzhou City, Jinzhou 121001, China

${ }^{\mathrm{c}}$ Key Laboratory of Cardiovascular and Cerebrovascular Drug Research of Liaoning Province, Jinzhou Medical University, Jinzhou 121001, China

*Correspondence to: Prof. Hongxin Wang, Department of Pharmacology, Jinzhou Medical University, No.40.Section 3, Songpo Road, Jinzhou City, Liaoning 121001, P.R. China. Tel:+0864164673466, Fax:0864163885335. Email: hongxinwang@lnmu.edu.cn 
Abstract Esculetin (6,7-dihydroxycoumarin) is a coumarin derivative extracted from natural plants and has been reported to have anti-cancer activity. However, the mechanism by which esculetin prevents human hepatic cancer cell growth is still largely unknown. In this study, we investigated the effect of esculetin on human hepatocellular carcinoma (HCC) SMMC-7721 cells and explored the cell signal mechanism. Our data indicated that esculetin induced apoptosis in SMMC-7721 cells, which were supported by DAPI staining and Annexin V/PI staining. Meanwhile, esculetin increased the activities of caspase-3 and caspase-9, promoted bax expression, decreased bcl-2 expression, and triggered collapse of mitochondrial membrane potential, cytochrome c release from mitochondria. In addition, the inactivation of IGF-1, PI3K and Akt was observed after esculetin administration. Furthermore, pretreatment with IGR-1 before esculetin administration abrogated the pro-apoptotic effects of esculetin. While PI3K inhibitor increased the pro-apoptotic effects of esculetin. These results indicated that esculetin induced the apoptosis of SMMC-7721 cells through IGF-1/PI3K/Akt-regulated mitochondrial dysfunction.

Keywords: Esculetin; Hepatocellular carcinoma SMMC-7721 cells; Apoptosis; IGF-1; Mitochondria

\section{Introduction}

Hepatocellular carcinoma (HCC) is one of the most prevalent malignancies around the world, having high morbidity and mortality. Currently, surgical resection and liver transplantation are the best options for treating HCC at the early stage 
(Belghiti and Fuks 2012; Gao et al. 2012). However, for most people diagnosed at the advanced stage, no effective therapeutic options are available because of its resistance and deleterious effects to chemotherapy and radiotherapy. Hence, a novel candidate selectively targeting carcinoma cells without compromising normal function is always the subject of investigation.

Esculetin (6,7-dihydroxycoumarin) is a coumarin derivative extracted from natural plants, such as Artemesia scoparia (Redstem Wormwood), Artemesia capillaris (Capillary Wormwood), and Ceratostigma willmottianum (Chinese Plumbago), and in the leaves of Citrus limonia (Chinese lemon) (Chang et al. 1996; Wang et al. 2002), that has been commonly used as a folk medicine. Esculetin has been reported to have various pharmacological and biochemical activities including anti-oxidant, anti-inflammatory and anti-proliferative and anti-obesity activity (Jeon et al. 2015; Karmase et al. 2013; Kim et al. 2008; Sulakhiya et al. 2016). These beneficial pharmacological and biochemical activities confer esculetin a potential therapeutic role in allergic asthma (Hongyan 2016), obesity (Yang et al. 2006), depression (Zhu et al. 2016) and diabetes (Prabakaran and Ashokkumar 2013). Although some herbal products have shown hepatotoxicity (Tarantino et al. 2009), more and more studies have focused on the anti-cancer activity of esculetin. These studies demonstrated that esculetin could induce the apoptosis of cancer cells, including human gastric carcinoma, colorectal cancer, malignant melanoma as well as breast carcinoma (Chang et al. 2016; Kim et al. 2015; Pan et al. 2015). The underlying mechanisms are involved in reactive oxygen species-mediated 
mitochondrial pathway and multiple signaling pathway et al. In addition, combination of esculetin with taxol could result in a further enhancement of apoptosis in human hepatoma HepG2 cells as compared to the treatment with taxol alone (Kuo et al. 2006). All these rusults suggested that esculetin has therapeutic potential for the treatment of human malignancies.

Insulin-like growth factor (IGF) axis plays a pivotal role in the development of normal cell growth and differentiation as well as progression of various cancers (Ali et al. 2014; Daqian et al. 2015; Durzynska 2014; Lindsey and Mohan 2016). It has been reported that IGF-1R knockout mice have only $50 \%$ body size as compared to the normal control mice (Baserga et al. 2003; Hartog et al. 2007). On the contrary, high circulating levels of IGF-1 are associated with increased risk of different types of cancers, largely depending on activation of the downstream cascade of the IGF axis (Sharon et al. 2015; Teng et al. 2016). After binding to IGF-1R, IGF-1 phosphorylates the downstream substrates, resulting in activation of the phosphatidylinositide 3-kinase/Akt (PI3K/Akt) and mitogen-activated protein kinase (MAPK) signaling cascades (Kong et al. 2016; Zhou et al. 2016). The over activation of PI3K/Akt and MAPK/ERK signaling pathways play a important role in increasing tumorigenesis, metastasis, and resistance to existing forms of cancer treatment through extensively phosphorylating apoptotic effector molecules, subsequently leading to cell proliferation and antiapoptosis (Appleman et al. 2012; Ju et al. 2015; Li et al. 2016). According to Jeon's report, the anti-proliferative effects of esculetin on oral squamous cell carcinoma were related to the inactivation of PI3K/Akt signaling pathway (Jeon 
et al. 2016). Our previous study indicated that esculetin could induce the apoptosis of hepatic carcinoma cells (Wang et al. 2015), but the mechanism by which esculetin is involved in the cross-link between the IGF-1/PI3K/Akt signaling pathway and mitochondrial function regulation is not well understood.

The findings of the present study demonstrate that esculetin suppresses the growth of human hepatic cancer SMMC-7721 cells, which might be related to the IGF-1 mediated mitochondrial dysfunction caused by the inactivation of the PI3K/Akt signaling pathway.

\section{Material and methods}

Materials

Esculetin was obtained from Nanjin Jingzhu Biotechnology Company (purity $>98 \%$; Nanjing, China); 3-(4,5-dimethylthiazol)-2,5-diphenyltetrazolium bromide (MTT) were obtained from Amresco (USA); The Annexin V-FITC apoptosis detection kit, DAPI and caspase-3/-9 were purchased from Beyotime Institute of Biotechnology; Antibodies specific for IGF-1 Bcl-2, Bax, PI3K, Akt, cytochrome c were purchased from abcam Technology; Antibodies specific for $\beta$-actin, GAPDH and VDAC were purchased from proteintech; IGF-1 and LY294002 were purchased from sigma company; RPMI 1640 medium and calf serum were purchased from Gibco.

Cell lines and cell culture

The human hepatic cancer SMMC-7721 cells were obtained from Type Culture Collection of the Chinese Academy of Sciences (Shanghai, China). Cells were 
cultured in RPMI 1640 medium containing 10\% fetal calf serum, 100 units $/ \mathrm{ml}$ streptomycin, and 100 units $/ \mathrm{ml}$ penicillin, in a humidified cell incubator at $37^{\circ} \mathrm{C}$ with an atmosphere of $5 \% \mathrm{CO}_{2}$.

Cell viability assay

SMMC-7721 cell viability was measured by the MTT method. Briefly, SMMC-7721 cells were seeded in 96-well plates at a density of $1.0 \times 10^{5}$ cells $/ \mathrm{ml}$ and cultured for $24 \mathrm{~h}$, then treated with $0,25,50,100,200$ or $300 \mu \mathrm{g} / \mathrm{mL}$ esculetin followed by incubation at $37^{\circ} \mathrm{C}$ for $24 \mathrm{~h}$. Thereafter, MTT was added to each well and the cells were incubated for an additional $4 \mathrm{~h}$ at $37^{\circ} \mathrm{C}$, then the formazan precipitate was dissolved in $150 \mu \mathrm{l}$ of DMSO, and the absorbance at $490 \mathrm{~nm}$ was measured using a microplate reader (BioTek Instruments, Inc.). Each test was repeated at least three times.

Apoptosis assays

The apoptotic rates were analyzed by flow cytometry using an annexin V-FITC apoptosis detection kit according to the manufacturer's instructions. Briefly, the cells were seeded in $25 \mathrm{~cm}^{2}$ flask and incubated with indicated drugs for $24 \mathrm{~h}$ before the cells were harvested. The cells were concentrated, and washed twice with cold PBS followed by staining with Annexin V-FITC using an assay kit. Data were analyzed using the Bioconsort software (USA). Apoptotic nuclear morphology was determined with DAPI staining. After SMMC-7721 Cells were incubated with different drugs for different times, the cells were fixed with $4 \%$ paraformaldehyde for $30 \mathrm{~min}$ at room temperature and then washed twice with PBS. Then cells were stained with the 
DNA-specific fluorescent dye DAPI for $10 \mathrm{~min}$ at $37^{\circ} \mathrm{C}$ and visualized using a fluorescence microscope (LEICA. DMI3000B)

Mitochondrial membrane potential (MMP)

Fluorogenic probe JC-1 was used to detect the MMP. JC-1 is a cationic dye that accumulates in mitochondria. Monomers of JC-1 dye fluoresce in the green range, whereas JC-1 aggregates fluoresce in the red range. Therefore, a decrease in red fluorescence intensity represents mitochondrial swelling. After SMMC-7721 Cells were incubated with different drugs for different times, the cells were wished with PBS, then incubated 20 minutes in the presence of $2 \mu \mathrm{M} \mathrm{JC}-1$ at $37^{\circ} \mathrm{C}$. Then the cells were washed with JC-1 buffer solution for twice. Thereafter, labeled cells were analyzed and quantified by fluorescence microscope (LEICA. DMI3000B).

Determination of caspase activity

Caspase-3 and caspase-9 activity was determined by colorimetric assay kits (Beyotime Institute of Biotechnology) according to the manufacturer's instructions. Caspase activity was determined by measuring changes in absorbance at $405 \mathrm{~nm}$ using microplate reader (BioTek Instruments, Inc.). All measurements were performed at least 3 times.

Mitochondrial extraction and Western blot analysis

Mitochondrial were extracted using mitochondria isolation kit. Briefly, the harvested cells were homogenated with mitochondria extraction reagent and centrifuged at $600 \mathrm{~g}, 4{ }^{0} \mathrm{C}$ for $5 \mathrm{~min}$. The supernatants were further centrifuged at $11,000 \mathrm{~g}, 4{ }^{0} \mathrm{C}$ for $10 \mathrm{~min}$ and the sediments were the mitochondria. The 
mitochondrial, cytoplasm and total protein concentration was determined by the Bradford method. After the samples was boiled for $5 \mathrm{~min}$, the protein samples were fractionated by SDS-PAGE and transferred to PVDF membrane (Millipore, Bedford, MA) using Semi dry transfer printing apparatus. The membranes were blocked with $1 \%$ bovine serum albumin solution at room temperature for $1.5 \mathrm{~h}$. Then the membranes were incubated with primary antibodies of IGF-1, p-PI3K, Akt, p-Akt, bcl-2, bax and cytochrome c at $4{ }^{\circ} \mathrm{C}$ overnight. The membranes were washed three times and then incubated with appropriate horseradish peroxidase-linked secondary antibodies at room temperature for $1.5 \mathrm{~h}$. Detection was performed with enhanced chemiluminescence reagents. The results were analyzed by the Quantity One software (Bio-Rad Laboratories, Hercules).

Statistic analysis

All data are expressed as mean \pm SD. SPSS 17.0 software was used to analyze all the data. Statistical analysis was performed using one-way ANOVA followed by Bonferroni's test. $p<0.05$ was considered statistically significant.

\section{Results}

Esculetin inhibits cell proliferation

To identify the therapeutic potential of esculetin, human SMMC-7721 cells were cultured with the indicated concentrations of esculetin for 24,48 or $72 \mathrm{~h}$. The cell viability was determined by MTT assay. Esculetin inhibited the growth of SMMC-7721 cells in a dose- and time-dependent manner (Fig. 1). The half-maximal 
inhibitory concentration $\left(\mathrm{IC}_{50}\right)$ for the SMMC-7721 cells at $72 \mathrm{~h}$ was $150 \mu \mathrm{g} / \mathrm{mL}$.

Esculetin induces apoptosis in human SMMC-7721 cells

To determine whether the cytotoxicity of esculetin was caused by the induction of apoptosis, DAPI staining assay were used. Pretreatment of cells with esculetin presented morphological features of early apoptosis, such as bright nuclear condensation and nuclear fragmentation. It appeared more frequently with increasing concentrations of esculetin. Then, Annexin V/PI staining was used to confirm these results. As shown in Fig. 2 after treatment with 75, 150 or $300 \mu \mathrm{g} / \mathrm{mL}$ esculetin for 24 $\mathrm{h}$, the percentage of apoptotic cells increased from $5.9 \%$ to $18.9 \%, 28.7 \%$ and $58.4 \%$ respectively. These results indicated that esculetin may exhibit the antitumor activity by inducing cell apoptosis.

Involvement of the mitochondrial pathway in esculetin-induced apoptosis

Mitochondrial dysfunction contributes to the process of cell apoptosis. The collapse of MMP is a maker for mitochondrial dysfunction in early stage of apoptosis. The results showed that control cells exhibited strong red JC-1 fluorescence, whereas pretreatment with esculetin exhibited a reduced level of red fluorescence and an increased level of green fluorescence, suggesting that esculetin administration could decrease the MMP (Fig. 3A). In addition, we examined the expressions of proteins involved in the mitochondria related apoptotic pathway. Esculetin administration increased the release of cytochrome c from mitochondria into cytoplasm, as well as 
reduced the bcl-2 expression and increased the bax expression, caspase- 9 and caspase-3 activity (Fig. 3B-G). All these data demonstrated that esculetin induced the apoptosis of SMMC-7721 cells in vitro probably through the mitochondrial apoptosis pathway.

Esculetin regulates IGF-1/PI3K/Akt pathway in SMMC-7721 cells

It has been reported that IGF-1/PI3K/Akt pathway is aberrantly activated in various types of cancers. We then investigated whether this pathway is implicated in the antitumor mechanism of esculetin. Western blot analysis showed that the protein expressions of IGF-1, p-PI3K and p-Akt were decreased after esculetin treatment, suggesting that esculetin administration could regulate IGF-1/PI3K/Akt pathway (Fig. 4).

Esculetin induces apoptosis via modulating IGF-1/PI3K/Akt related mitochondrial pathway

To determine whether esculetin induced apoptosis is associated with modulating IGF-1/PI3K/Akt pathway related mitochondrial pathway, the effect of $50 \mu \mathrm{M}$ IGF-1 alone or in combination with esculetin on SMMC-7721 cells was evaluated in this study. The results showed that IGF-1 treatment alone increased cell proliferation, inhibited apoptoctic protein bax expression, and increased antiapoptotic protein bcl-2 expression, which were related with activated IGF-1/PI3K/Akt pathway. What is more, pretreatment cell with IGF-1 before $150 \mu \mathrm{g} / \mathrm{mL}$ esculetin reversed the pro-apoptotic 
effects of esculetin, as evidenced by increased cell proliferation and bcl-2/bax ratio, improvement of nuclear morphological changes and MMP, reduction of cytochrome c from mitochondria to cytoplasm (Fig. 5). Further more, western blot analysis showed that the antagonistic action of IGF-1 to esculetin was related with moderate activation of IGF-1/PI3K/Akt pathway (Fig. 6). In addition, to further identify the role of Akt in esculetin induced growth inhibition, we employed the inhibitor LY294002 to inhibit Akt. Combination treatment with esculetin and LY294002 could further enhance the apoptotic effect of esculetin. These results together suggested that pro-apoptotic effects of esculetin were acted with IGF-1/PI3K/Akt pathway related mitochondrial pathway.

\section{Discussion}

As a considerably valuable source for novel chemotherapeutic agents, the traditional Chinese medicine products remain one of the best reservoirs of new molecules. Esculetin, one of the traditional Chinese medicine products, has been reported to have potential antitumor activity in various human cancers. In our previous study, we have demonstrated that esculetin could inhibit human hepatic cancer growth both in vito and in vitro, but the underlying mechanism was not well studied. In the present study, we showed that esculetin induces mitochondria-mediated apoptosis in human SMMC-7721 cells, as evidenced by a decrease of mitochondrial potential, increase of bax/bcl-2 expression ratio and cytochrome c translocation from mitochondria to cytosol as well as activation of caspase-3 and caspase-9. In addition, the pro-apopototic effect of esculetin was related with inactivation of IGF-1/PI3K/Akt 
signaling pathways. Furthermore, the IGF-1 reversed the pro-apoptotic effects of esculetin, while the PI3K inhibitor contributed the pro-apoptotic effects of esculetin.

The IGF-1 pathway has been implicated in the etiology of several epithelial malignancies, including liver, breast, colon, prostate, and gynecologic cancers (Werner and Bruchim 2009), and interference with IGF-1R function appears to be potentially effective therapeutic strategy for cancer. For example, Simvastatin was reported to induce bile duct cancer cells apoptosis by suppressing IGF-1R activity (Lee et al. 2016). However, there is controversy regarding the effect of IGF-1 pathway in HCC since IGF-1 is mainly produced in the liver. IGF-1 level reflects hepatic function and is inversely correlated with the severity of background chronic liver disease (Assy et al. 2008; Lorenzo-Zuniga et al. 2007). In contrast with these, some literatures reported that the baseline mRNA levels of IGF-1 in HCC patients was higher than in normal person (Karabulut et al. 2014), and the expression of IGF-1R was higher in hepatocellular carcinoma cell lines compared with normal human hepotocytes, and prcropodophyllin, a highly selective inhibitor of IGF-1R, was reported to has pro-apoptotic effect of hepatocellular carcinoma cell through a caspase-depended mitochontrial pathway (E et al. 2013). In addition, retrospective study showed that higher IGF-1 expression in adjacent non-neoplastic liver than in tumor, and it was correlated with significantly poorer survival after resection of $\mathrm{HCC}$ (Chun et al. 2014). Anyway, in our present study, we showed that IGF-1 promoted SMMC-7721 cells proliferation, and the pro-apoptotic effect of esculetin was related with down-regulated IGF-1 expression, which was in line with some previous studies. 
When IGF-1 binds to IGF-1R, the tyrosine kinase leads to phosphorylation of downstream receptor substrate, which results in activation of its downstream signaling pathways, including the Ras/MAPK and PI3K/Akt pathways. Activated PI3K/Akt can arrest cells in the G0/G1 phase of the cell cycle via modulation of mammalian target of rapamycin (mToR) and regulating cyclin D1(Hashemolhosseini et al. 1998). Beside, activated Akt extensively phosphorylate apoptotic effector molecules, such as Bcl-2, Mcl-1, Bim, Bad, caspase-9 and many others. More and more evidences suggesting that bcl-2 family of proteins, which include antiapoptotic and proapoptotic factors plays a pivotal role in the control and regulation of mitochondrial apoptotic pathway (Shan and Fan 2016; Xu et al. 2014). The proapoptotic protein bax induces the permeabilization of the outer mitochondrial membrane, leads to cytochrome c release from mitochondrial to cytoplasm, and subsequently activates caspase-3. On the other hand, bcl-2 prevents mitochondrial permeabilization and inhibites apoptosis (Childs et al. 2002). Based on our study, esculetin inhitied SMMC-7721 cells proliferation, induced cell apoptosis and mitochondrial permeabilization, increased caspase-3 and caspase-9 activity, bax/bcl-2 ration and cytochrome c release from mitochondrial. These results demonstrated that esculetin induced SMMC-7721 cells apoptosis via mitochondrial pathway. However, further study indicated that the proapoptotic effects of esculetin were closed accompanied with inactivation of IGF-1/PI3K/Akt sinaling pathway. Of note, IGF-1 attenuated the proapoptotic effects of esculetin, while LY294002, the inhibitor of PI3K increased the effects of esculetin, which further illustrated molecular mechanism of proapoptotic effect of esculetin on SMMC-7721 
cells. However, the association of inflammation with HCC has been previously suggested, inflammatory factors such as TNF- $\alpha$ and IL-6 not only contribute to the progression of HCC, TNF- $\alpha$ also promotes insulin resistance by decreasing tyrosine kynase activity of the insulin receptor and consequently reducing insulin activity (Hotamisligil et al. 1996), TNF- $\alpha$ and interleukin inhibitors have been used for treatment of active ankylosing apondylitis (Betts et al. 2016). IGF-1 is increased in patients with type 2 diabetes mellitus, the increased IGF-1 is responsible for the developing of cancer, and it has been suggested that non-alcoholic fatty liver disease (NAFLD) as a new criterion to define metabolic syndrome (MetS) (Tarantino and Finelli 2013), so the effects of esculetin on inflammatory factors and NAFLD should be investigated in the future study.

In summary, the current study demonstrated that esculetin exerts strong antitumor activity in human SMMC-7721 cells with the potential molecular mechanisms of apoptosis induction through IGF-1/PI3K/Akt related mitochondria pathway. Therefore, our study indicates that esculetin might be a promising therapeutic agent against human hepatic cancer.

\section{Conflict of interest}

The authors declare that there are no conflicts of interest.

\section{Acknowledgements}

This work was supported by Natural Science Foundation of Liaoning Province (No. 
2015020347)

\section{References:}

Ali, M., Kumar, A., and Pandey, B.N. 2014. Thorium induced cytoproliferative effect in human liver cell HepG2: role of insulin-like growth factor 1 receptor and downstream signaling. Chem. Biol. Interact. 211: 29-35. doi: 10.1016/j.cbi.2014.01.006.

Appleman, V.A., Ahronian, L.G., Cai, J., Klimstra, D.S., and Lewis, B.C. 2012. KRAS(G12D)- and BRAF(V600E)-induced transformation of murine pancreatic epithelial cells requires MEK/ERK-stimulated IGF1R signaling. Mol. Cancer Res. 10(9): 1228-1239. doi: 10.1158/1541-7786.MCR-12-0340-T.

Assy, N., Pruzansky, Y., Gaitini, D., Shen Orr, Z., Hochberg, Z., and Baruch, Y. 2008. Growth hormone-stimulated IGF-1 generation in cirrhosis reflects hepatocellular dysfunction. J. Hepatol. 49(1): 34-42. doi: 10.1016/j.jhep.2008.02.013.

Baserga, R., Peruzzi, F., and Reiss, K. 2003. The IGF-1 receptor in cancer biology. Int. J. Cancer, 107(6): 873-877. doi: 10.1002/ijc. 11487.

Belghiti, J., and Fuks, D. 2012. Liver resection and transplantation in hepatocellular carcinoma. Liver Cancer, 1(2): 71-82. doi: 10.1159/000342403.

Betts, K.A., Griffith, J., Song, Y., Mittal, M., Joshi, A., Wu, E.Q., and Ganguli, A. 2016. Network Meta-Analysis and Cost Per Responder of Tumor Necrosis Factor-alpha and Interleukin Inhibitors in the Treatment of Active Ankylosing Spondylitis. Rheumatol. Ther. 3(2): 323-336 doi: 10.1007/s40744-016-0038-y.

Chang, H.T., Chou, C.T., Lin, Y.S., Shieh, P., Kuo, D.H., Jan, C.R., and Liang, W.Z. 2016. Esculetin, a natural coumarin compound, evokes $\mathrm{Ca}(2+)$ movement and activation of $\mathrm{Ca}(2+)$-associated mitochondrial apoptotic pathways that involved cell cycle arrest in ZR-75-1 human breast cancer cells. Tumour Biol. 37(4): 4665-4678. doi: 10.1007/s13277-015-4286-1.

Chang, W.S., Lin, C.C., Chuang, S.C., and Chiang, H.C. 1996. Superoxide anion scavenging effect of coumarins. Am. J. Chin. Med. 24(1): 11-17. doi: 10.1142/S0192415X96000037.

Childs, A.C., Phaneuf, S.L., Dirks, A.J., Phillips, T., and Leeuwenburgh, C. 2002. Doxorubicin treatment in vivo causes cytochrome $\mathrm{C}$ release and cardiomyocyte apoptosis, as well as increased mitochondrial efficiency, superoxide dismutase activity, and Bcl-2:Bax ratio. Cancer Res. 62(16): 4592-4598.

Chun, Y.S., Huang, M., Rink, L., and Von Mehren, M. 2014. Expression levels of insulin-like growth factors and receptors in hepatocellular carcinoma: a retrospective study. World J. Surg. Oncol. 12: 231. doi: 10.1186/1477-7819-12-231.

Daqian, W., Chuandong, W., Xinhua, Q., Songtao, A., and Kerong, D. 2015. Chimaphilin inhibits proliferation and induces apoptosis in multidrug resistant osteosarcoma cell lines through insulin-like growth factor-I receptor (IGF-IR) signaling. Chem. Biol. Interact. 237: 25-30. doi: 10.1016/j.cbi.2015.05.008.

Durzynska, J. 2014. IGF axis and other factors in HPV-related and HPV-unrelated carcinogenesis (review). Oncol. Rep. 32(6): 2295-2306. doi: 10.3892/or.2014.3505.

E, C., Li, J., Shao, D., Zhang, D., Pan, Y., Chen, L., and Zhang, X. 2013. The insulin-like growth factor-I receptor inhibitor picropodophyllin-induced selective apoptosis of hepatocellular carcinoma cell through a caspase-dependent mitochondrial pathway. Oncol. Res. 21(2): 103-110. doi: 
10.3727/096504013X13808175127324.

Gao, J.J., Song, P.P., Tamura, S., Hasegawa, K., Sugawara, Y., Kokudo, N., Uchida, K., Orii, R., Qi, F.H., Dong, J.H., and Tang, W. 2012. Standardization of perioperative management on hepato-biliary-pancreatic surgery. Drug. Discov. Ther. 6(2): 108-111.

Hartog, H., Wesseling, J., Boezen, H.M., and van der Graaf, W.T. 2007. The insulin-like growth factor 1 receptor in cancer: old focus, new future. Eur. J. Cancer, 43(13): 1895-1904. doi: 10.1016/j.ejca.2007.05.021.

Hashemolhosseini, S., Nagamine, Y., Morley, S.J., Desrivieres, S., Mercep, L., and Ferrari, S. 1998. Rapamycin inhibition of the G1 to $\mathrm{S}$ transition is mediated by effects on cyclin D1 mRNA and protein stability. J. Biol. Chem. 273(23): 14424-14429.

Hongyan, L. 2016. Esculetin Attenuates Th2 and Th17 Responses in an Ovalbumin-Induced Asthmatic Mouse Model. Inflammation, 39(2): 735-743. doi: 10.1007/s10753-015-0300-4.

Hotamisligil, G.S., Peraldi, P., Budavari, A., Ellis, R., White, M.F., and Spiegelman, B.M. 1996. IRS-1-mediated inhibition of insulin receptor tyrosine kinase activity in TNF-alpha- and obesity-induced insulin resistance. Science, 271(5249): 665-668.

Jeon, Y.J., Cho, J.H., Lee, S.Y., Choi, Y.H., Park, H., Jung, S., Shim, J.H., and Chae, J.I. 2016. Esculetin Induces Apoptosis Through EGFR/PI3K/Akt Signaling Pathway and Nucleophosmin Relocalization. J. Cell. Biochem. 117(5): 1210-1221. doi: 10.1002/jcb.25404.

Jeon, Y.J., Jang, J.Y., Shim, J.H., Myung, P.K., and Chae, J.I. 2015. Esculetin, a Coumarin Derivative, Exhibits Anti-proliferative and Pro-apoptotic Activity in G361 Human Malignant Melanoma. J. Cancer Prev. 20(2): 106-112. doi: 10.15430/JCP.2015.20.2.106.

Ju, D.T., Liao, H.E., Shibu, M.A., Ho, T.J., Padma, V.V., Tsai, F.J., Chung, L.C., Day, C.H., Lin, C.C., and Huang, C.Y. 2015. Nerve Regeneration Potential of Protocatechuic Acid in RSC96 Schwann Cells by Induction of Cellular Proliferation and Migration through IGF-IR-PI3K-Akt Signaling. Chin. J. Physiol. 58(6): 412-419. doi: 10.4077/CJP.2015.BAD340.

Karabulut, S., Duranyildiz, D., Tas, F., Gezer, U., Akyuz, F., Serilmez, M., Ozgur, E., Yasasever, C.T., Vatansever, S., and Aykan, N.F. 2014. Clinical significance of serum circulating insulin-like growth factor-1 (IGF-1) mRNA in hepatocellular carcinoma. Tumour Biol. 35(3): 2729-2739. doi: 10.1007/s13277-013-1360-4.

Karmase, A., Birari, R., and Bhutani, K.K. 2013. Evaluation of anti-obesity effect of Aegle marmelos leaves. Phytomedicine, 20(10): 805-812. doi: 10.1016/j.phymed.2013.03.014.

Kim, A.D., Madduma Hewage, S.R., Piao, M.J., Kang, K.A., Cho, S.J., and Hyun, J.W. 2015. Esculetin induces apoptosis in human colon cancer cells by inducing endoplasmic reticulum stress. Cell. Biochem. Funct. 33(7): 487-494. doi: 10.1002/cbf.3146.

Kim, S.H., Kang, K.A., Zhang, R., Piao, M.J., Ko, D.O., Wang, Z.H., Chae, S.W., Kang, S.S., Lee, K.H., Kang, H.K., Kang, H.W., and Hyun, J.W. 2008. Protective effect of esculetin against oxidative stress-induced cell damage via scavenging reactive oxygen species. Acta Pharmacol. Sin. 29(11): 1319-1326. doi: 10.1111/j.1745-7254.2008.00878.x.

Kong, D., Gong, L., Arnold, E., Shanmugam, S., Fort, P.E., Gardner, T.W., and Abcouwer, S.F. 2016. Insulin-like growth factor 1 rescues R28 retinal neurons from apoptotic death through ERK-mediated BimEL phosphorylation independent of Akt. Exp. Eye Res. 151: 82-95. doi: 10.1016/j.exer.2016.08.002.

Kuo, H.C., Lee, H.J., Hu, C.C., Shun, H.I., and Tseng, T.H. 2006. Enhancement of esculetin on Taxol-induced apoptosis in human hepatoma HepG2 cells. Toxicol. Appl. Pharmacol. 210(1-2): 
55-62. doi: 10.1016/j.taap.2005.06.020.

Lee, J., Hong, E.M., Jang, J.A., Park, S.W., Koh, D.H., Choi, M.H., Jang, H.J., and Kae, S.H. 2016. Simvastatin Induces Apoptosis and Suppresses Insulin-Like Growth Factor 1 Receptor in Bile Duct Cancer Cells. Gut Liver, 10(2): 310-317. doi: 10.5009/gn115195.

Li, J., Teng, Y., Liu, S., Wang, Z., Chen, Y., Zhang, Y., Xi, S., Xu, S., Wang, R., and Zou, X. 2016. Cinnamaldehyde affects the biological behavior of human colorectal cancer cells and induces apoptosis via inhibition of the PI3K/Akt signaling pathway. Oncol. Rep. 35(3): 1501-1510. doi: 10.3892/or.2015.4493.

Lindsey, R.C., and Mohan, S. 2016. Skeletal effects of growth hormone and insulin-like growth factor-I therapy. Mol. Cell. Endocrinol. 432: 44-55. doi: 10.1016/j.mce.2015.09.017.

Lorenzo-Zuniga, V., Bartoli, R., Masnou, H., Montoliu, S., Morillas, R.M., and Planas, R. 2007. Serum concentrations of insulin-like growth factor-I (igf-I) as a marker of liver fibrosis in patients with chronic hepatitis C. Dig. Dis. Sci. 52(11): 3245-3250. doi: 10.1007/s10620-006-9437-1.

Pan, H., Wang, B.H., Lv, W., Jiang, Y., and He, L. 2015. Esculetin induces apoptosis in human gastric cancer cells through a cyclophilin D-mediated mitochondrial permeability transition pore associated with ROS. Chem. Biol. Interact. 242: 51-60. doi: 10.1016/j.cbi.2015.09.015.

Prabakaran, D., and Ashokkumar, N. 2013. Protective effect of esculetin on hyperglycemia-mediated oxidative damage in the hepatic and renal tissues of experimental diabetic rats. Biochimie, 95(2): 366-373. doi: 10.1016/j.biochi.2012.10.008.

Shan, M., and Fan, T.J. 2016. Cytotoxicity of carteolol to human corneal epithelial cells by inducing apoptosis via triggering the Bcl-2 family protein-mediated mitochondrial pro-apoptotic pathway. Toxicol. In Vitro, 35: 36-42. doi: 10.1016/j.tiv.2016.05.008.

Sharon, C., Baranwal, S., Patel, N.J., Rodriguez-Agudo, D., Pandak, W.M., Majumdar, A.P., Krystal, G., and Patel, B.B. 2015. Inhibition of insulin-like growth factor receptor/AKT/mammalian target of rapamycin axis targets colorectal cancer stem cells by attenuating mevalonate-isoprenoid pathway in vitro and in vivo. Oncotarget, 6(17): 15332-15347. doi: 10.18632/oncotarget.3684.

Sulakhiya, K., Keshavlal, G.P., Bezbaruah, B.B., Dwivedi, S., Gurjar, S.S., Munde, N., Jangra, A., Lahkar, M., and Gogoi, R. 2016. Lipopolysaccharide induced anxiety- and depressive-like behaviour in mice are prevented by chronic pre-treatment of esculetin. Neurosci. Lett. 611: 106-111. doi: 10.1016/j.neulet.2015.11.031.

Tarantino, G., and Finelli, C. 2013. What about non-alcoholic fatty liver disease as a new criterion to define metabolic syndrome? World J. Gastroenterol. 19(22): 3375-3384. doi: 10.3748/wjg.v19.i22.3375.

Tarantino, G., Pezzullo, M.G., di Minno, M.N., Milone, F., Pezzullo, L.S., Milone, M., and Capone, D. 2009. Drug-induced liver injury due to "natural products" used for weight loss: a case report. World J. Gastroenterol. 15(19): 2414-2417.

Teng, J.A., Wu, S.G., Chen, J.X., Li, Q., Peng, F., Zhu, Z., Qin, J., and He, Z.Y. 2016. The Activation of ERK1/2 and JNK MAPK Signaling by Insulin/IGF-1 Is Responsible for the Development of Colon Cancer with Type 2 Diabetes Mellitus. PLoS One, 11(2): e0149822. doi: 10.1371/journal.pone.0149822.

Wang, C.J., Hsieh, Y.J., Chu, C.Y., Lin, Y.L., and Tseng, T.H. 2002. Inhibition of cell cycle progression in human leukemia HL-60 cells by esculetin. Cancer Lett.183(2): 163-168.

Wang, J., Lu, M.L., Dai, H.L., Zhang, S.P., Wang, H.X., and Wei, N. 2015. Esculetin, a coumarin derivative, exerts in vitro and in vivo antiproliferative activity against hepatocellular carcinoma by 
initiating a mitochondrial-dependent apoptosis pathway. Braz. J. Med. Biol. Res. 48(3): 245-253. doi: 10.1590/1414-431X20144074.

Werner, H., and Bruchim, I. 2009. The insulin-like growth factor-I receptor as an oncogene. Arch. Physiol. Biochem. 115(2): 58-71. doi: 10.1080/13813450902783106.

Xu, S., Sui, G., Yuan, L., and Zou, Z. 2014. Expression of programmed cell death 5 protein inhibits progression of lung carcinoma in vitro and in vivo via the mitochondrial apoptotic pathway. Mol. Med. Rep. 10(4): 2059-2064. doi: 10.3892/mmr.2014.2454.

Yang, J.Y., Della-Fera, M.A., Hartzell, D.L., Nelson-Dooley, C., Hausman, D.B., and Baile, C.A. 2006. Esculetin induces apoptosis and inhibits adipogenesis in 3T3-L1 cells. Obesity (Silver Spring), 14(10): 1691-1699. doi: 10.1038/oby.2006.194.

Zhou, Y., Zeng, C., Li, X., Wu, P.L., Yin, L., Yu, X.L., Zhou, Y.F., and Xue, Q. 2016. IGF-I stimulates ERbeta and aromatase expression via IGF1R/PI3K/AKT-mediated transcriptional activation in endometriosis. J. Mol. Med. (Berl.) 94(8): 887-897. doi: 10.1007/s00109-016-1396-1.

Zhu, L., Nang, C., Luo, F., Pan, H., Zhang, K., Liu, J., Zhou, R., Gao, J., Chang, X., He, H., Qiu, Y., Wang, J., Long, H., Liu, Y., and Yan, T. 2016. Esculetin attenuates lipopolysaccharide (LPS)-induced neuroinflammatory processes and depressive-like behavior in mice. Physiol. Behav. 163: 184-192. doi: 10.1016/j.physbeh.2016.04.051. 


\section{Figure legends}

Fig. 1. Effects of esculetin on cell viability. (A) Chemical structure of esculetin. (B) Effects of esculetin on cell viability. Cell viability was detected by the MTT assay. SMMC-7721 cells were treated with indicated concentrations of esculetin for $24 \mathrm{~h}$, $48 \mathrm{~h}$ and $72 \mathrm{~h}$. Data represents the mean $\pm \mathrm{SD}$ of three independent experiments.

Fig. 2. Esculetin induces apoptosis in human SMMC-7721 cells. (A) Apoptotic body formation was observed by fluorescence microscopy after DAPI staining. (B) Apoptosis detected in SMMC-7721 cells after treatment with indicated esculetin for $24 \mathrm{~h}$ by annexin V-FITC/PI double staining assay. (C) Bar graphs show that the cell apoptotic rates increase in human SMMC-7721 cells after treatment with esculetin for $24 \mathrm{~h}$ in a dose-dependent manner. Data represents the mean $\pm \mathrm{SD}$ of three independent experiments $(* p<0.05$ versus control).

Fig. 3. Involvement of the mitochondrial pathway in esculetin-induced apoptosis.

(A) Cells were stained with JC-1 and mitochondrial membrane potential was assessed by fluorescence microscope. (B-C) Bcl-2 and bax expressions assessed by western blot. (D) Caspase-3 and caspase-9 activity assessed by commercial kits. (E-G) Mitochondrial and cytosolic cytochrome c expressions assessed by western blot. Data represents the mean $\pm \mathrm{SD}$ of three independent experiments $\left({ }^{*} p<0.05\right.$ versus control). 
Fig. 4. The effects of esculetin on IGF-1/PI3K/Akt pathway in SMMC-7721 cells. Cells were treated with different concentrations of esculetin for $24 \mathrm{~h}$, and IGF-1, p-PI3K, Akt and p-Akt expressions were determined by western blot. Data represents the mean $\pm \mathrm{SD}$ of three independent experiments $\left({ }^{*} p<0.05\right.$ versus control).

Fig. 5. The effects of IGF-1 and LY294002 on esculetin induced cell apoptosis and caspase activity. (A) Cells were stained with JC-1. (B) Cells were stained with DAPI and apoptotic body formation was observed by fluorescence microscopy. (C) Bar graphs show the cell apoptosis assessed by annexin V-FITC/PI double staining. (D) Caspase- 3 and caspase- 9 activity assessed by commercial kits. Data represents the mean $\pm \mathrm{SD}$ of three independent experiments $(* p<0.05$ versus control).

Fig. 6. Effects of different drugs on expressions of apoptosis related proteins. (A-B) protein expression assessed by western blot. (C) IGF-1 protein expression. (D) changes in mitochondrial and cytosolic cytochorme c expression. (E) Bcl-2 and bax expression changes. (F) Different drugs on p-PI3K and p-Akt expression. Data represents the mean $\pm \mathrm{SD}$ of three independent experiments $\left({ }^{*} p<0.05\right.$ versus control). 
A<smiles>O=c1ccc2cc(O)c(O)cc2o1</smiles>

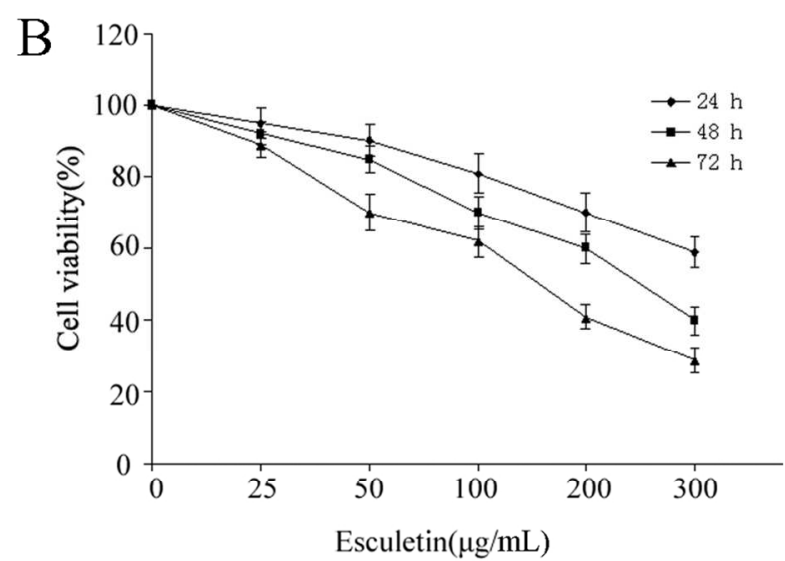

Fig. 1. Effects of esculetin on cell viability. (A) Chemical structure of esculetin. (B) Effects of esculetin on cell viability. Cell viability was detected by the MTT assay. SMMC-7721 cells were treated with indicated concentrations of esculetin for $24 \mathrm{~h}, 48 \mathrm{~h}$ and $72 \mathrm{~h}$. Data represents the mean \pm SD of three independent experiments.

$118 \times 59 \mathrm{~mm}(300 \times 300 \mathrm{DPI})$ 


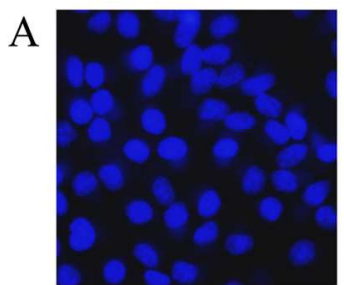

Control

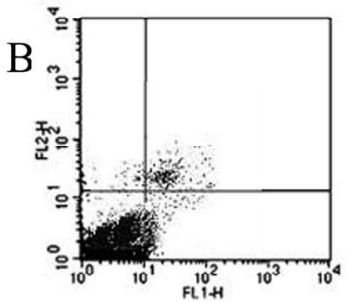

Control

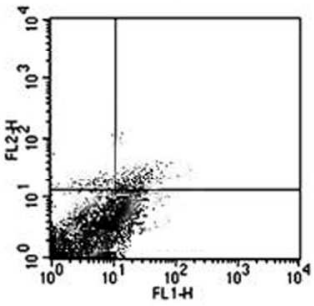

Esculetin(150)

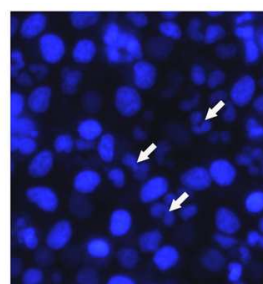

Esculetin(75)

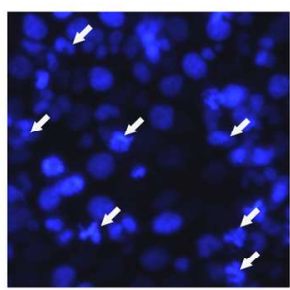

Esculetin(150)

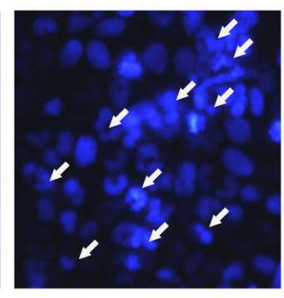

Esculetin(300)

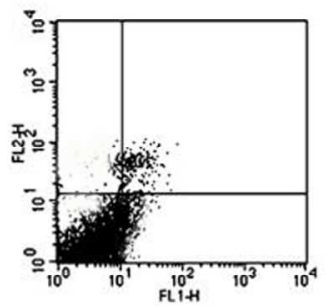

Esculetin(75)

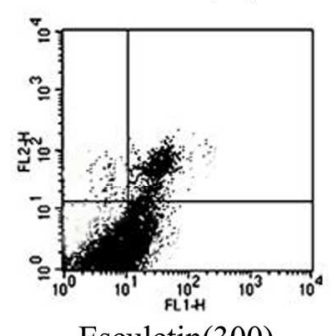

$\mathrm{C}$

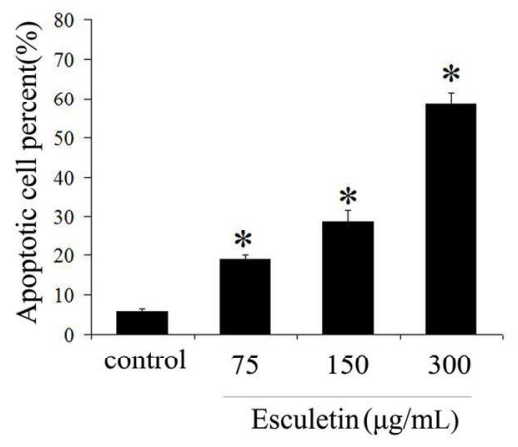

Esculetin(300)

Fig. 2. Esculetin induces apoptosis in human SMMC-7721 cells. (A) Apoptotic body formation was observed by fluorescence microscopy after DAPI staining. (B) Apoptosis detected in SMMC-7721 cells after treatment with indicated esculetin for $24 \mathrm{~h}$ by annexin V-FITC/PI double staining assay. (C) Bar graphs show that the cell apoptotic rates increase in human SMMC-7721 cells after treatment with esculetin for $24 \mathrm{~h}$ in a dosedependent manner. Data represents the mean \pm SD of three independent experiments $(* p<0.05$ versus control). 


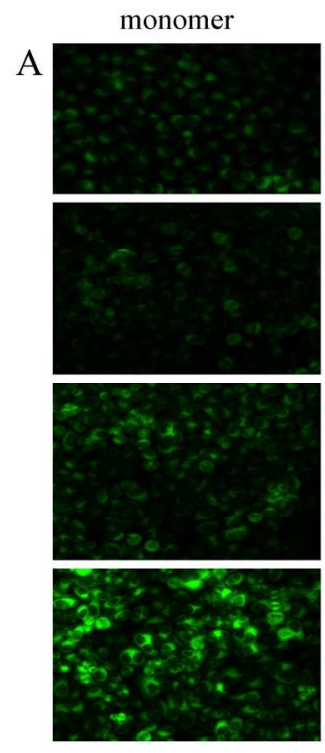

B

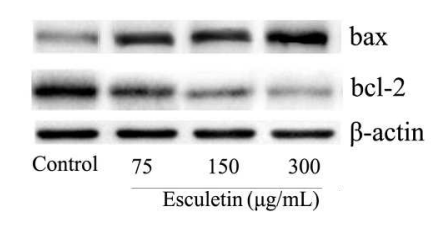

$\mathrm{E}$

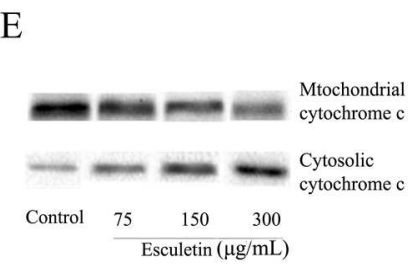

aggregate
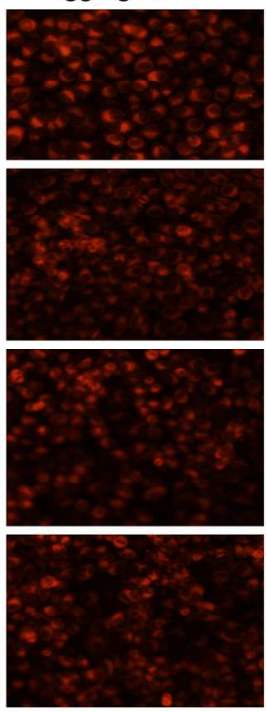

C

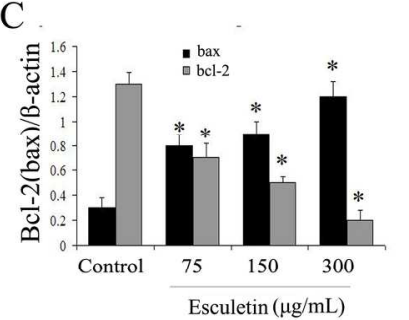

$\mathrm{F}$

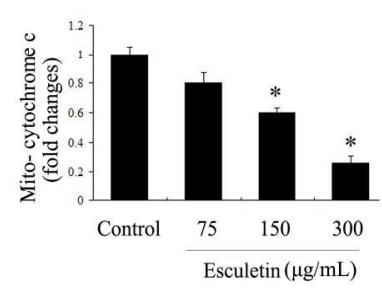

overlay

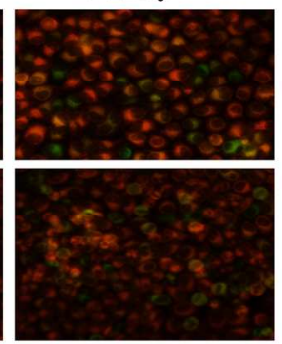

Control

Esculetin $(75 \mu \mathrm{g} / \mathrm{mL})$

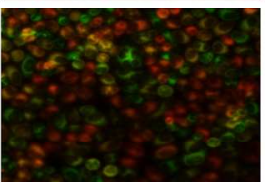

Esculetin $(150 \mu \mathrm{g} / \mathrm{mL})$

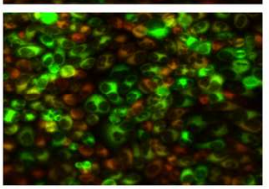

Esculetin(300 $\mu \mathrm{g} / \mathrm{mL})$

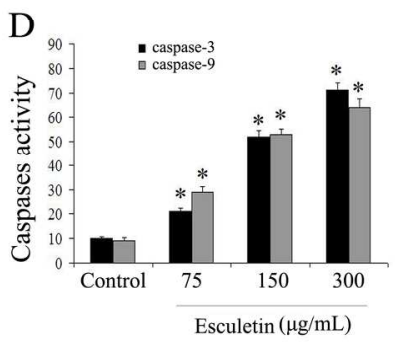

G

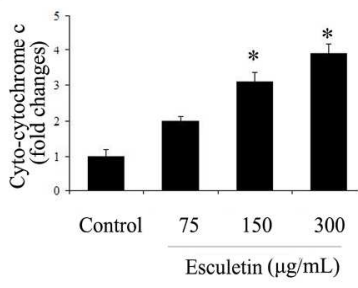

Fig. 3. Involvement of the mitochondrial pathway in esculetin-induced apoptosis. (A) Cells were stained with $\mathrm{JC}-1$ and mitochondrial membrane potential was assessed by fluorescence microscope. (B-C) Bcl-2 and bax expressions assessed by western blot. (D) Caspase- 3 and caspase- 9 activity assessed by commercial kits. (E-G) Mitochondrial and cytosolic cytochrome c expressions assessed by western blot. Data represents the mean \pm SD of three independent experiments $(* p<0.05$ versus control).

$$
191 \times 216 \mathrm{~mm} \text { ( } 300 \times 300 \text { DPI) }
$$



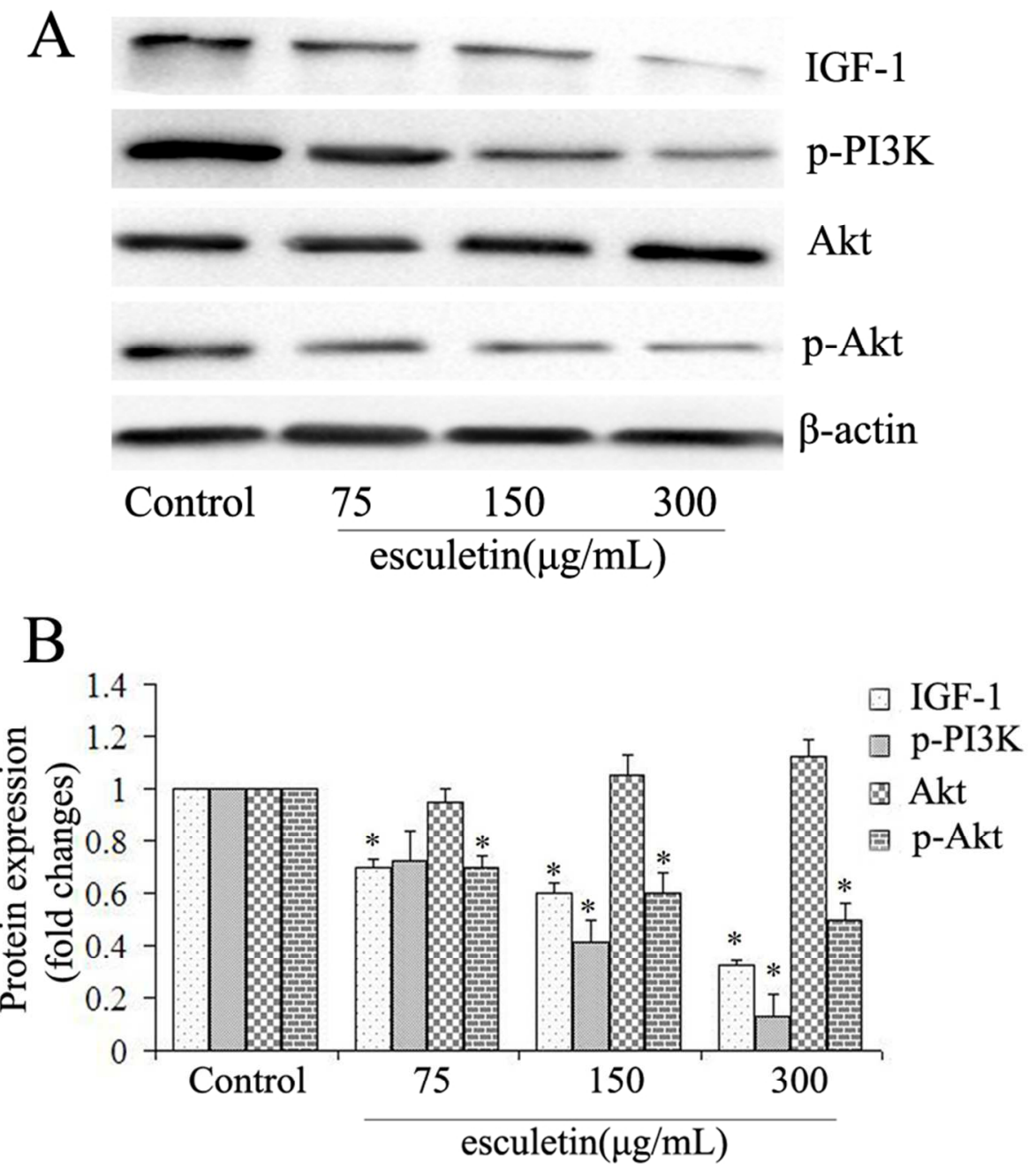

Fig. 4. The effects of esculetin on IGF-1/PI3K/Akt pathway in SMMC-7721 cells. Cells were treated with different concentrations of esculetin for 24h, and IGF-1, p-PI3K, Akt and p-Akt expressions were determined by western blot. Data represents the mean \pm SD of three independent experiments $(* p<0.05$ versus control).

$85 \times 90 \mathrm{~mm}(300 \times 300 \mathrm{DPI})$ 


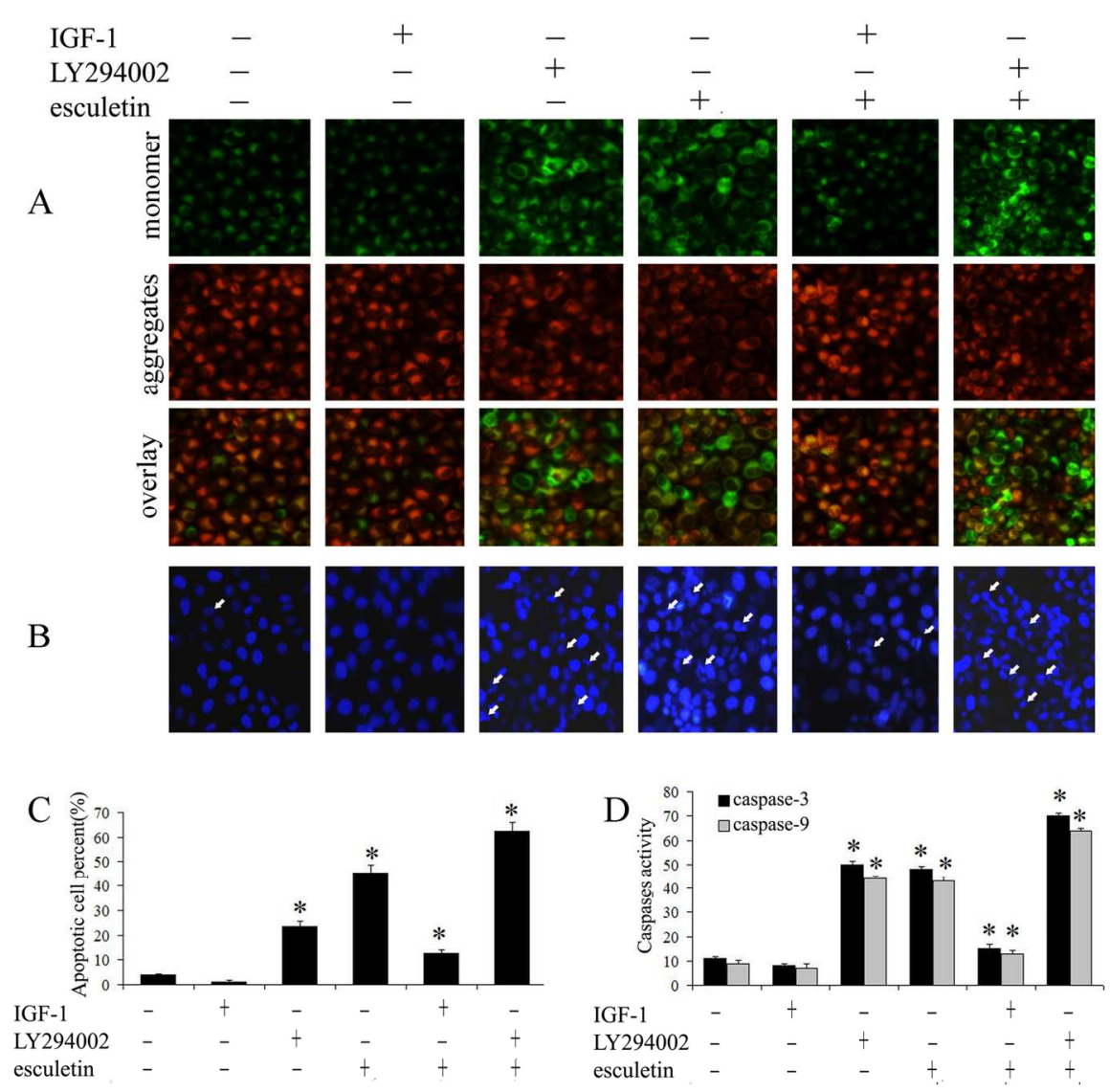

Fig. 5. The effects of IGF-1 and LY294002 on esculetin induced cell apoptosis and caspase activity. (A) Cells were stained with JC-1. (B) Cells were stained with DAPI and apoptotic body formation was observed by fluorescence microscopy. (C) Bar graphs show the cell apoptosis assessed by annexin V-FITC/PI double staining. (D) Caspase-3 and caspase- 9 activity assessed by commercial kits. Data represents the mean \pm SD of three independent experiments $(* p<0.05$ versus control).

$149 \times 132 \mathrm{~mm}(300 \times 300 \mathrm{DPI})$ 

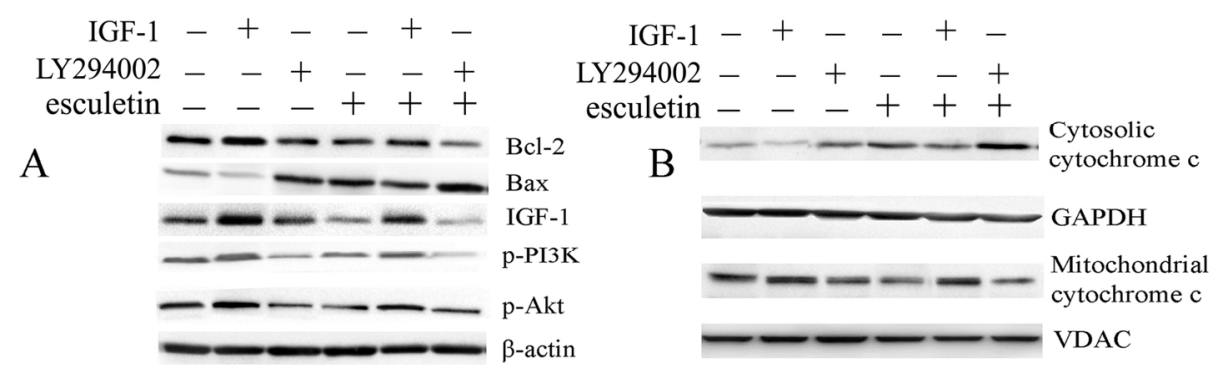

$\mathrm{C}$

$\mathrm{D}$
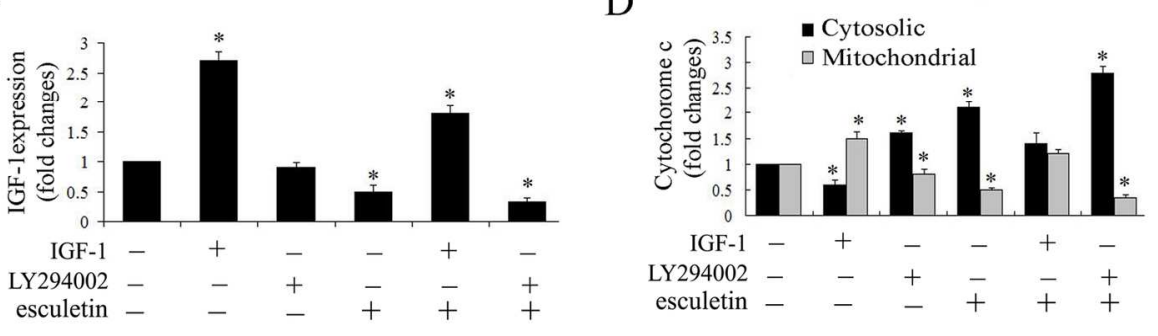

E
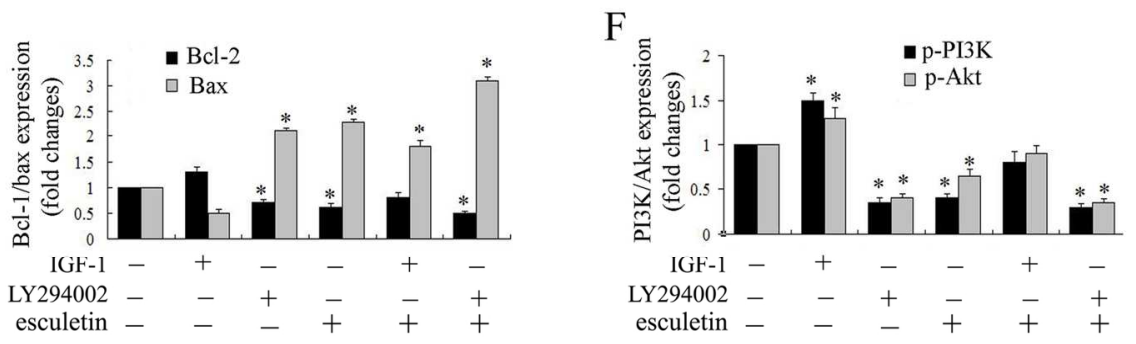

Fig. 6. Effects of different drugs on expressions of apoptosis related proteins. (A-B) protein expression assessed by western blot. (C) IGF-1 protein expression. (D) changes in mitochondrial and cytosolic cytochorme c expression. (E) Bcl-2 and bax expression changes. (F) Different drugs on p-PI3K and p-Akt expression. Data represents the mean \pm SD of three independent experiments ( ${ }^{*} p<0.05$ versus control).

$$
149 \times 132 \mathrm{~mm} \text { ( } 300 \times 300 \text { DPI) }
$$

